\title{
NUMERICAL SOLUTIONS TO INTEGRAL EQUATIONS EQUIVALENT TO DIFFERENTIAL EQUATIONS WITH FRACTIONAL TIME
}

\author{
BARTOSZ BANDROWSKI* , ANNA KARCZEWSKA*, PIOTR ROZMEJ ** \\ * Faculty of Mathematics, Computer Science and Econometrics \\ University of Zielona Góra, ul. Szafrana 4a, 65-516 Zielona Góra, Poland \\ email: \{B.Bandrowski, A.Karczewska\}@wmie.uz.zgora.pl \\ ${ }^{* *}$ Institute of Physics \\ University of Zielona Góra, ul. Szafrana 4a, 65-516 Zielona Góra, Poland \\ email: P.Rozmej@if.uz.zgora.pl
}

\begin{abstract}
This paper presents an approximate method of solving the fractional (in the time variable) equation which describes the processes lying between heat and wave behavior. The approximation consists in the application of a finite subspace of an infinite basis in the time variable (Galerkin method) and discretization in space variables. In the final step, a large-scale system of linear equations with a non-symmetric matrix is solved with the use of the iterative GMRES method.
\end{abstract}

Keywords: fractional equations, Galerkin method, anomalous diffusion.

\section{Introduction}

Several physical phenomena show anomalous transport properties. Generally, diffusive properties are classified as normal if their variance grows linearly in time and as anomalous if the variance growth is different from the linear one. In recent years, there has been an increasing interest in dynamical processes occuring in systems exhibiting anomalous diffusive behavior. Such systems range from physics and chemistry to biology and medicine (Meltzer and Klafter, 2000). Dispersion in complex plasmas (Ratynskaia et al., 2006), self-diffusion of surfactant molecules (Gambin et al., 2005), light in a cold atomic cloud (Labeyrie et al., 2003) and donor-acceptor electron pairs within a protein (Kou and Sunney Xie, 2004) are examples of the more recent experimental evidences. Several papers (Goychuk et al., 2006; Heinsalu et al., 2006; Heinsalu et al., 2009) consider the fractional Fokker-Planck equation both in an analytical and a numerical approach. The fractional time approach is considered also in control theory, see, e.g., (Kaczorek, 2008; Guermah et al., 2008). An interesting numerical approach to solving partial differential equations of fractional orders in space was presented in (Ciesielski and Leszczyński, 2006).
In most of applications related to fractional differential or fractional integro-differential equations, the numerical methods are limited to $1+1$ (time+space) dimensions. Our paper presents a different method for solving a fractional integro-differential equation which can handle more dimensional cases within a good approximation. The method is limited to cases when the initial condition $u(x, 0)$ is smooth enough with respect to space variables $x$. In such cases it works also for $(1+2)$ and $(1+3)$ dimensions.

We consider the following Volterra equation:

$$
u(x, t)=u(x, 0)+\int_{0}^{t} a(t-s) \Delta u(x, s) \mathrm{d} s,
$$

where $x \in \mathbb{R}^{d}, t>0, a(t)=t^{\alpha-1} / \Gamma(\alpha), \Gamma$ is the gamma function, $\alpha \in[1,2]$ and $\Delta$ is the Laplace operator.

Because of the form of the kernel function $a$, the integral in Eqn. (1) is a Riemann-Liouville fractional integral operator (Bazhlekova, 2001),

$$
J_{t}^{\alpha} f(t):=\int_{0}^{t} \frac{1}{\Gamma(\alpha)}(t-s)^{\alpha-1} f(s) \mathrm{d} s .
$$


Applying the Caputo fractional derivative operator to (1), one obtains a fractional differential equation,

$$
\frac{\partial^{\alpha}}{\partial t^{\alpha}} u(x, t)=\Delta u(x, t)
$$

where $\alpha \in[1,2], x \in \mathbb{R}^{d}, t>0$ and $\Delta$ is the Laplace operator.

For particular cases when $\alpha=1$ and $\alpha=2$, taking appropriate initial conditions, (2) is the heat and the wave equation, respectively. For $\alpha \in(1,2)$, (2) interpolates the heat and the wave equations.

Equation (1) was discussed by Fujita (1990) and Schneider and Wyss (1989). Fujita found the analytical solution $u(x, t)$ to (1) in terms of fundamental solutions using Mittag-Leffler functions. Schneider and Wyss applied the Green function method and obtained the analytical form of the solution in terms of Wright functions (which are related to Mittag-Leffler functions). Both approaches are limited to a $(1+1)$-dimensional case and practically non-computable.

\section{Construction of the numerical solution}

The numerical approach described below follows in general that described in (Rozmej and Karczewska, 2005).

Let there be given a set of real orthonormal functions $\left\{\phi_{j}: j=1,2, \ldots, \infty\right\}$ on the interval $[0, t]$, spanning a Hilbert space $H$ with an inner product

$$
\langle f, g\rangle:=\int_{0}^{t} f(\tau) g(\tau) W(\tau) \mathrm{d} \tau
$$

where $W$ is a weight function.

An approximate solution to (1) is considered an element of the subspace $H_{n}$ spanned by the first $n$ basis functions $\left\{\phi_{k}: k=1,2, \ldots, n\right\}$,

$$
u_{n}(x, t)=\sum_{k=1}^{n} c_{k}(x) \phi_{k}(t)
$$

Inserting (3) into (1), one obtains

$$
\begin{aligned}
& u_{n}(x, t) \\
& \quad=u(x, 0)+\int_{0}^{t} a(t-s) \Delta u_{n}(x, s) \mathrm{d} s+\epsilon_{n}(x, t),
\end{aligned}
$$

where $\epsilon_{n}$ denotes the approximation error. From (3) and (4), we get the following form of the error function:

$$
\begin{aligned}
\epsilon_{n}(x, t)= & \sum_{k=1}^{n} c_{k}(x) \phi_{k}(t)-u(x, 0) \\
& -\int_{0}^{t} a(t-s) \sum_{k=1}^{n} \frac{\mathrm{d}^{2}}{\mathrm{~d} x^{2}} c_{k}(x) \phi_{k}(s) \mathrm{d} s .
\end{aligned}
$$

Definition 1. The Galerkin approximation of the solution to Eqn. (1) is a function $u_{n} \in H_{n}$ such that $\epsilon_{n} \perp H_{n}$, i.e.,

$$
\left\langle\epsilon_{n}(x, t), \phi_{k}(t)\right\rangle=0, \quad k=1,2, \ldots, n .
$$

From Definition 1 and (5), we obtain

$$
\begin{aligned}
0= & \int_{0}^{t}\left[\sum_{k=1}^{n} c_{k}(x) \phi_{k}(\tau)\right] \phi_{j}(\tau) W(\tau) \mathrm{d} \tau \\
& -\int_{0}^{t} u(x, 0) \phi_{j}(\tau) W(\tau) \mathrm{d} \tau \\
- & \int_{0}^{t}\left[\int_{0}^{\tau} a(\tau-s) \sum_{k=1}^{n} \frac{\mathrm{d}^{2}}{\mathrm{~d} x^{2}} c_{k}(x) \phi_{k}(s) \mathrm{d} s\right] \\
& \times \phi_{j}(\tau) W(\tau) \mathrm{d} \tau .
\end{aligned}
$$

for $j=1,2, \ldots, n$, which means that

$$
\begin{aligned}
\int_{0}^{t} u(x, 0) \phi_{j}(\tau) W(\tau) \mathrm{d} \tau \\
=\int_{0}^{t}\left[\sum_{k=1}^{n} c_{k}(x) \phi_{k}(\tau)\right] \phi_{j}(\tau) W(\tau) \mathrm{d} \tau \\
-\int_{0}^{t}\left[\int_{0}^{\tau} a(\tau-s) \sum_{k=1}^{n} \frac{\mathrm{d}^{2}}{\mathrm{~d} x^{2}} c_{k}(x) \phi_{k}(s) \mathrm{d} s\right] \\
\quad \times \phi_{j}(\tau) W(\tau) \mathrm{d} \tau .
\end{aligned}
$$

The above equations may be written in a shortened form,

$$
g_{j}(x)=c_{j}(x)-\sum_{k=1}^{n} a_{j k} \frac{\mathrm{d}^{2}}{\mathrm{~d} x^{2}} c_{k}(x),
$$

where

$$
a_{j k}=\int_{0}^{t}\left[\int_{0}^{\tau} a(\tau-s) \phi_{k}(s) \mathrm{d} s\right] \phi_{j}(\tau) W(\tau) \mathrm{d} \tau
$$

and

$$
g_{j}(x)=u(x, 0) \int_{0}^{t} \phi_{j}(\tau) W(\tau) \mathrm{d} \tau
$$

$j=1,2, \ldots, n$. In general, $a_{j k} \neq a_{k j}$.

Equations (6) describe a set of coupled differential equations for the coefficient functions $c_{k}(x), k=$ $1,2, \ldots, n$ determining the approximate solution (3).

To solve the set (6), we use standard, centered threepoint finite difference approximation to the second derivative (Laplacian). 
In one spatial dimension one obtains (6) as

$$
\begin{aligned}
g_{j}\left(x_{i}\right)= & c_{j}\left(x_{i}\right)+\frac{1}{h^{2}} \sum_{k=1}^{n} a_{j k} \\
& \times\left[-c_{k}\left(x_{i-1}\right)+2 c_{k}\left(x_{i}\right)-c_{k}\left(x_{i+1}\right)\right],
\end{aligned}
$$

where there holds $x_{i}-x_{i-1}=h, j=1,2, \ldots, n$, and $i=1,2, \ldots, m$.

For two spatial dimensions and a homogenous grid, (6) can be written as

$$
\begin{aligned}
g_{j}\left(x_{i}, y_{l}\right)= & c_{j}\left(x_{i}, y_{l}\right)+\frac{1}{h^{2}} \sum_{k=1}^{n} a_{j k} \\
\times & {\left[-c_{k}\left(x_{i-1}, y_{l}\right)-c_{k}\left(x_{i}, y_{l-1}\right)\right.} \\
& +4 c_{k}\left(x_{i}, y_{l}\right)-c_{k}\left(x_{i+1}, y_{l}\right) \\
& \left.-c_{k}\left(x_{i}, y_{l+1}\right)\right]
\end{aligned}
$$

where $x_{i}-x_{i-1}=y_{l}-y_{l-1}=h$ and $j=1,2, \ldots, n$, $i=1,2, \ldots, m, l=1,2, \ldots, m$. form

The sets (7) and (8) can be presented in the matrix

$$
g=\mathcal{A} c,
$$

where the vectors $g, c$ and the matrix $\mathcal{A}$ have a block structure,

$$
\begin{gathered}
g=\left(\begin{array}{c}
G_{1} \\
G_{2} \\
\vdots \\
G_{n}
\end{array}\right), \quad c=\left(\begin{array}{c}
C_{1} \\
C_{2} \\
\vdots \\
C_{n}
\end{array}\right), \\
\mathcal{A}=\left(\begin{array}{ccc}
{\left[A_{11}\right]} & \cdots & {\left[A_{1 n}\right]} \\
{\left[A_{21}\right]} & \cdots & {\left[A_{2 n}\right]} \\
\vdots & \ddots & \vdots \\
{\left[A_{n 1}\right]} & \cdots & {\left[A_{n n}\right]}
\end{array}\right) .
\end{gathered}
$$

In the one-dimensional case (7), for (10) we have

$$
G_{j}^{T}=\left(g_{j}\left(x_{1}\right), g_{j}\left(x_{2}\right), \ldots, g_{j}\left(x_{m}\right)\right)
$$

and

$$
C_{j}^{T}=\left(c_{j}\left(x_{1}\right), c_{j}\left(x_{2}\right), \ldots, c_{j}\left(x_{m}\right)\right),
$$

where $G_{j}^{T}$ and $C_{j}^{T}$ stand for the transposes of vectors $G_{j}$ and $C_{j}$, respectively. Blocks $\left[A_{j k}\right]$ have the following structure:

$$
\left[A_{j k}\right]=\left(\begin{array}{cccccc}
\mu_{j k} & \eta_{j k} & 0 & \cdots & 0 & \theta_{j k} \\
\eta_{j k} & \mu_{j k} & \eta_{j k} & \cdots & 0 & 0 \\
0 & \eta_{j k} & \mu_{j k} & \cdots & 0 & 0 \\
\vdots & \vdots & \vdots & \ddots & \vdots & \vdots \\
0 & 0 & 0 & \cdots & \mu_{j k} & \eta_{j k} \\
\theta_{j k} & 0 & 0 & \cdots & \eta_{j k} & \mu_{j k}
\end{array}\right)_{m \times m},
$$

where

$$
\begin{gathered}
\mu_{j k}=\delta_{j k}+\frac{2}{h^{2}} a_{j k}, \\
\eta_{j k}=-\frac{1}{h^{2}} a_{j k},
\end{gathered}
$$

and

$$
\theta_{j k}= \begin{cases}-\frac{1}{h^{2}} a_{j k} & \text { for periodic boundary conditions, } \\ 0 & \text { for closed boundary conditions. }\end{cases}
$$

The vectors $g$ and $c$ are $n m$-dimensional, and the matrix $\mathcal{A}$ is $n m \times n m$-dimensional. Moreover, the matrix $\mathcal{A}$ is sparse with at most $n^{2}(3 m-2)$ (closed boundary conditions) or $3 n^{2} m$ (periodic boundary conditions) non-zero elements. In the two-dimensional case (8), we have

$$
\begin{aligned}
G_{j}^{T}= & \left(g_{j}\left(x_{1}, y_{1}\right), g_{j}\left(x_{1}, y_{2}\right), \ldots, g_{j}\left(x_{1}, y_{m}\right),\right. \\
& \left.g_{j}\left(x_{2}, y_{1}\right), g_{j}\left(x_{2}, y_{2}\right), \ldots, g_{j}\left(x_{m}, y_{m}\right)\right)
\end{aligned}
$$

and

$$
\begin{aligned}
C_{j}^{T}= & \left(c_{j}\left(x_{1}, y_{1}\right), c_{j}\left(x_{1}, y_{2}\right), \ldots, c_{j}\left(x_{1}, y_{m}\right)\right. \\
& \left.c_{j}\left(x_{2}, y_{1}\right), c_{j}\left(x_{2}, y_{2}\right), \ldots, c_{j}\left(x_{m}, y_{m}\right)\right)
\end{aligned}
$$

Each of blocks $\left[A_{j k}\right]$ is a matrix in the form of

$$
\left[A_{j k}\right]=\left(\begin{array}{ccccccc}
\left(\alpha_{j k}\right)\left(\beta_{j k}\right) & (0) & \cdots & (0) & (0) & \left(\gamma_{j k}\right) \\
\left(\beta_{j k}\right) & \left(\alpha_{j k}\right) & \left(\beta_{j k}\right) & \cdots & (0) & (0) & (0) \\
(0) & \left(\beta_{j k}\right) & \left(\alpha_{j k}\right) & \cdots & (0) & (0) & (0) \\
\vdots & \vdots & \vdots & \ddots & \vdots & \vdots & \vdots \\
(0) & (0) & (0) & \cdots & \left(\alpha_{j k}\right) & \left(\beta_{j k}\right) & (0) \\
(0) & (0) & (0) & \cdots & \left(\beta_{j k}\right)\left(\alpha_{j k}\right) & \left(\beta_{j k}\right) \\
\left(\gamma_{j k}\right) & (0) & (0) & \cdots & (0) & \left(\beta_{j k}\right) & \left(\alpha_{j k}\right)
\end{array}\right)_{m \times m}
$$

which consists of smaller blocks, where

$$
\left(\gamma_{j k}\right)= \begin{cases}\left(\beta_{j k}\right) & \text { for periodic boundary conditions } \\ (0) & \text { for closed boundary conditions. }\end{cases}
$$

Block (0) is an $m \times m$-dimensional zero matrix, block $\left(\alpha_{j k}\right)$ is in the form of

$$
\left(\alpha_{j k}\right)=\left(\begin{array}{cccccc}
\mu_{j k} & \eta_{j k} & 0 & \cdots & 0 & \theta_{j k} \\
\eta_{j k} & \mu_{j k} & \eta_{j k} & \cdots & 0 & 0 \\
0 & \eta_{j k} & \mu_{j k} & \cdots & 0 & 0 \\
\vdots & \vdots & \vdots & \ddots & \vdots & \vdots \\
0 & 0 & 0 & \cdots & \mu_{j k} & \eta_{j k} \\
\theta_{j k} & 0 & 0 & \cdots & \eta_{j k} & \mu_{j k}
\end{array}\right)_{m \times m}
$$

where

$$
\begin{gathered}
\mu_{j k}=\delta_{j k}+\frac{4}{h^{2}} a_{j k}, \\
\eta_{j k}=-\frac{1}{h^{2}} a_{j k},
\end{gathered}
$$


$\theta_{j k}= \begin{cases}-\frac{1}{h^{2}} a_{j k} & \text { for periodic boundary conditions, } \\ 0 & \text { for closed boundary conditions, }\end{cases}$

Block $\left(\beta_{j k}\right)$ is in the diagonal form,

$\left(\beta_{j k}\right)=\left(\begin{array}{ccccccc}\frac{-1}{h^{2}} a_{j k} & 0 & 0 & \cdots & 0 & 0 & 0 \\ 0 & \frac{-1}{h^{2}} a_{j k} & 0 & \cdots & 0 & 0 & 0 \\ 0 & 0 & \frac{-1}{h^{2}} a_{j k} & \cdots & 0 & 0 & 0 \\ \vdots & \vdots & \vdots & \ddots & \vdots & \vdots & \vdots \\ 0 & 0 & 0 & \cdots & \frac{-1}{h^{2}} a_{j k} & 0 & 0 \\ 0 & 0 & 0 & \cdots & 0 & \frac{-1}{h^{2}} a_{j k} & 0 \\ 0 & 0 & 0 & \cdots & 0 & 0 & \frac{-1}{h^{2}} a_{j k}\end{array}\right)_{m \times m .}$

The vectors $g$ and $c$ are $n m^{2}$-dimensional, and the matrix $\mathcal{A}$ is $n m^{2} \times n m^{2}$-dimensional. As in the previous case, the matrix $\mathcal{A}$ is sparse. It has at most $n^{2}(5 m-4) m$ (closed boundary conditions) or $5 n^{2} m^{2}$ (periodic boundary conditions) non-zero elements. As basis functions $\left\{\phi_{j}: j=1,2, \ldots, \infty\right\}$ one can choose any polynomials orthogonal with respect to some weight function $W$.

In our numerical scheme, we use Legendre polynomials, which are orthogonal on the interval $[-1,1]$ with respect to the weight function $W \equiv 1$. We obtain orthonormality on the interval $[0, t]$ by scaling the argument of the function and using a normalisation factor, i.e.,

$$
\phi_{j}(x)=\sqrt{\frac{2 k-1}{t}} P_{k-1}\left(\frac{2 x}{t}-1\right),
$$

where $\left\{P_{k}: k=0,1,2, \ldots, \infty\right\}$ are Legendre polynomials.

The matrix $\mathcal{A}$ of the linear system 9 is sparse, which, taken in conjunction with the large matrix size, suggests the use of iterative methods for solving linear systems. Morover, the matrix $\mathcal{A}$ is non-symmetric. Our numerical results were obtained with two different iterative methods. The first one, GMRES (Generalized Minimal Resiudal method) (Saad and Schultz, 1986), approximates the solution by the vector with a minimal residual in a Krylov subspace found with the use of the Arnoldi iteration. The second one, Bi-CGSTAB (BiConjugate Gradient Stabilized method) (Van der Vorst, 1992), was developed to solve non-symmetric linear systems. It avoids irregular convergence patterns of CGS (Conjugate Gradient Squared method) (Barrett et al., 1994). In both methods a suitable preconditioning is necessary. For our purposes, GMRES appeared more efficient. It was converging faster and usually required fewer iterations than the Bi-CGSTAB method.

For three spatial dimensions, vectors $g$ and $c$ in (9) become $\mathrm{nm}^{3}$-dimensional. The matrix $\mathcal{A}$ preserves its block nested structure (10) but with one more level of nesting. The number of the non-zero elements of the matrix $\mathcal{A}$ reaches the order of $7 n^{2} m^{3}$. Therefore, the numerical solution for the $d=3$ case requires much more computer power than for one and two-dimensional cases.

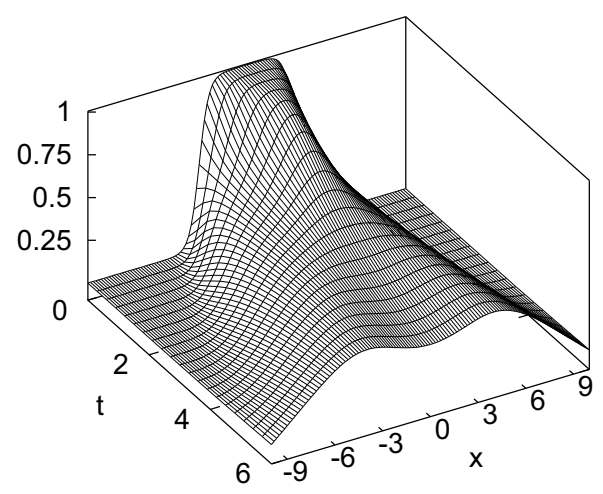

Fig. 1. Numerical solutions to the Volterra equation 11 in one spatial dimension: $\alpha=1.5, t \in[0,6]$, closed boundary conditions.

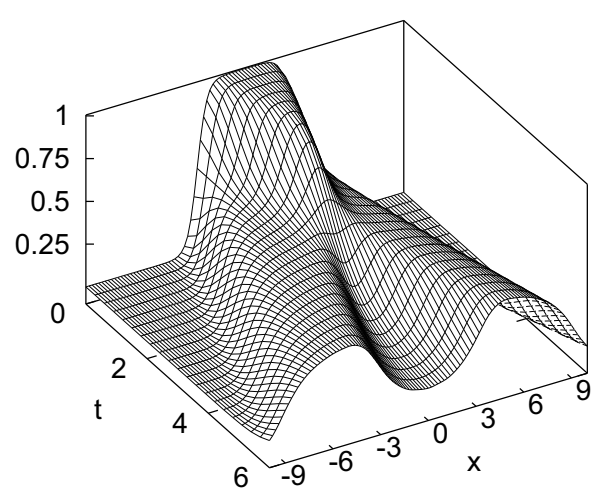

Fig. 2. Numerical solutions to the Volterra equation (1) in one spatial dimension: $\alpha=1.85, t \in[0,6]$, closed boundary conditions.

\section{Examples of numerical results}

Analytical solutions to fractional equations are of great importance but they are hardly computable. In most cases, to obtain a solution to a particular problem one has to apply suitable numerical methods. In (Rozmej and Karczewska, 2005) we showed that for the cases when analytical solutions are known in terms of elementary functions (1+1-dimensional cases with $\alpha=1$ and $\alpha=2$ ), our approximate numerical solution reproduces the analytical ones with high accuracy.

We present numerical results to the Volterra equation (1) in three cases: in one spatial dimension with closed boundary conditions (Figs. 1 and 2) and in two spatial dimensions with both closed (Figs. 3 and 4) and periodic boundary conditions (Figs. 5 and 6).

For the presentation of the results, we choose as initial conditions the Fermi distribution:

$$
u(\vec{r}, 0)=\frac{1}{1+\exp \left(\frac{r-r_{0}}{a}\right)},
$$


where $r=\left(\sum_{i=1}^{d} x_{i}^{2}\right)^{1 / 2}$ and $d$ is the space dimension. The constants in (12) are taken as $r_{0}=3, a=0.3$.

In Table 1, parameters $m$ (the number of grid points in space variables) and $n$ (the number of basis functions) used to obtain numerical results are presented. In principle, when the basis of orthonormal functions is richer, the numerical results are more precise. A similar argument applies to the number of grid points taken in the problem discretization. However, the growing size of matrices (as the result of the increase in the number of grid points and basis functions) results in an incrementation in the number of computer operations and in the accumulation of round-off errors. Comparing Figs. 3 and 4 or Figs. 5 and 6, one can notice that for the 1+2-dimensional case the increase in $\alpha$ changes the time evolution from diffussion-like behavior to wave-like one. The comparison of the results obtained with the same $\alpha$ (for instance, Figs. 3] and 5 or Figs. 4 and 6) shows the influence of 'waves' incoming to the given space cell from the neighbours.

The method used in the paper has some limitations. Numerical approximation of the second derivatives (Laplacian) can be good enough when the initial condition $u(x, 0)$ is a relatively smooth function, i.e., it varies unrapidly with respect to space variables within a range of grid points.

Figures 1 and 2 show the time evolution for the fractional time derivative of the order $\alpha=1.5$ and $\alpha=1.85$, respectively. One can clearly see that the system evolves in a way which exhibits wave motion with a diffusive character. When $\alpha$ varies towards 1, one obtains more diffusionlike evolution, whereas for $\alpha$ closer to 2, wave-like evolution becomes dominant. The closed (or free) boundary conditions mean that the initial system can in principle evolve to infinity in the space variable, where initially $u(x, 0)=0$. In the presented figures, however, the solution is cut off to the range of the space variable where the solutions differs from zero substantially.

\section{Approximation errors}

As mentioned earlier, the analytic form of the solution $u(x, t)$ is known only for one space dimension (Fujita, 1990; Schneider and Wyss, 1989). However, the analytical solutions for cases different from $\alpha=1$ and $\alpha=2$, given in terms of Mittag-Leffler functions, are practically non-computable. To estimate the quality of the numerical solutions, several tests were performed. For $d=1$ and

Table 1. Parameters.

\begin{tabular}{|c|c|c|}
\hline Case & Grid size & Basis functions \\
\hline \hline$d=1$ & 121 & 21 \\
\hline$d=2$, closed b. c. & $101 \times 101$ & 21 \\
\hline$d=2$, periodic b. c. & $71 \times 71$ & 21 \\
\hline
\end{tabular}
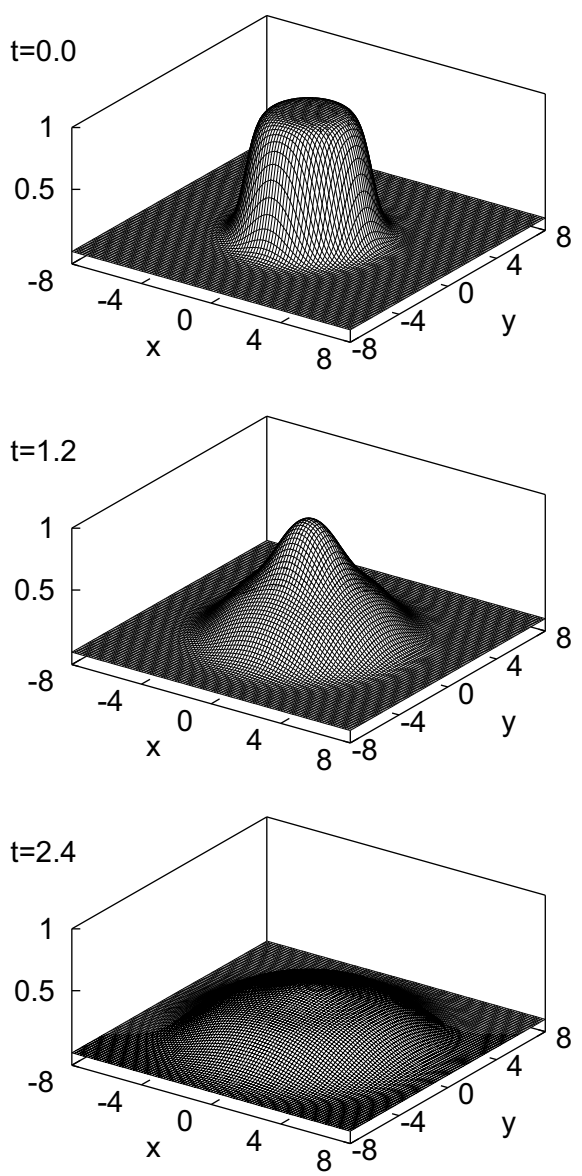

Fig. 3. Numerical solutions to the Volterra equation (1) in two spatial dimensions at chosen time steps: $\alpha=1.5$, closed boundary conditions.

$\alpha=1$ and $\alpha=1$, we can compare the analytical solution with the numerical one obtained from (3) and (9). As an error estimate, we define the maximum absolute value of the difference between the exact analytical solution and the approximate numerical one,

$$
\Delta u_{n, m}(t)=\max \left|u_{n, m}^{\mathrm{anal}}\left(x_{i}, t\right)-u_{n, m}^{\mathrm{num}}\left(x_{i}, t\right)\right|_{i=1}^{m},
$$

where the maximum is taken over all grid points $x_{i}$. For $d=1$ and $\alpha=1$ and $2, n>20, m>100, t \leq 6$, the error estimate $\Delta u_{n, m}(t)$ was always less than $10^{-5}-10^{-6}$.

For cases when we have to rely only on the approximate numerical solutions $(1<\alpha<2)$, we procede in a different way. One can expect that when the number of basis functions ( $n$ in (3) ) or the size of grid $(m)$ increases, better approximation of the solution is obtained. In order to show that trend, we define the absolute value of the difference between the two approximate solutions taken for 

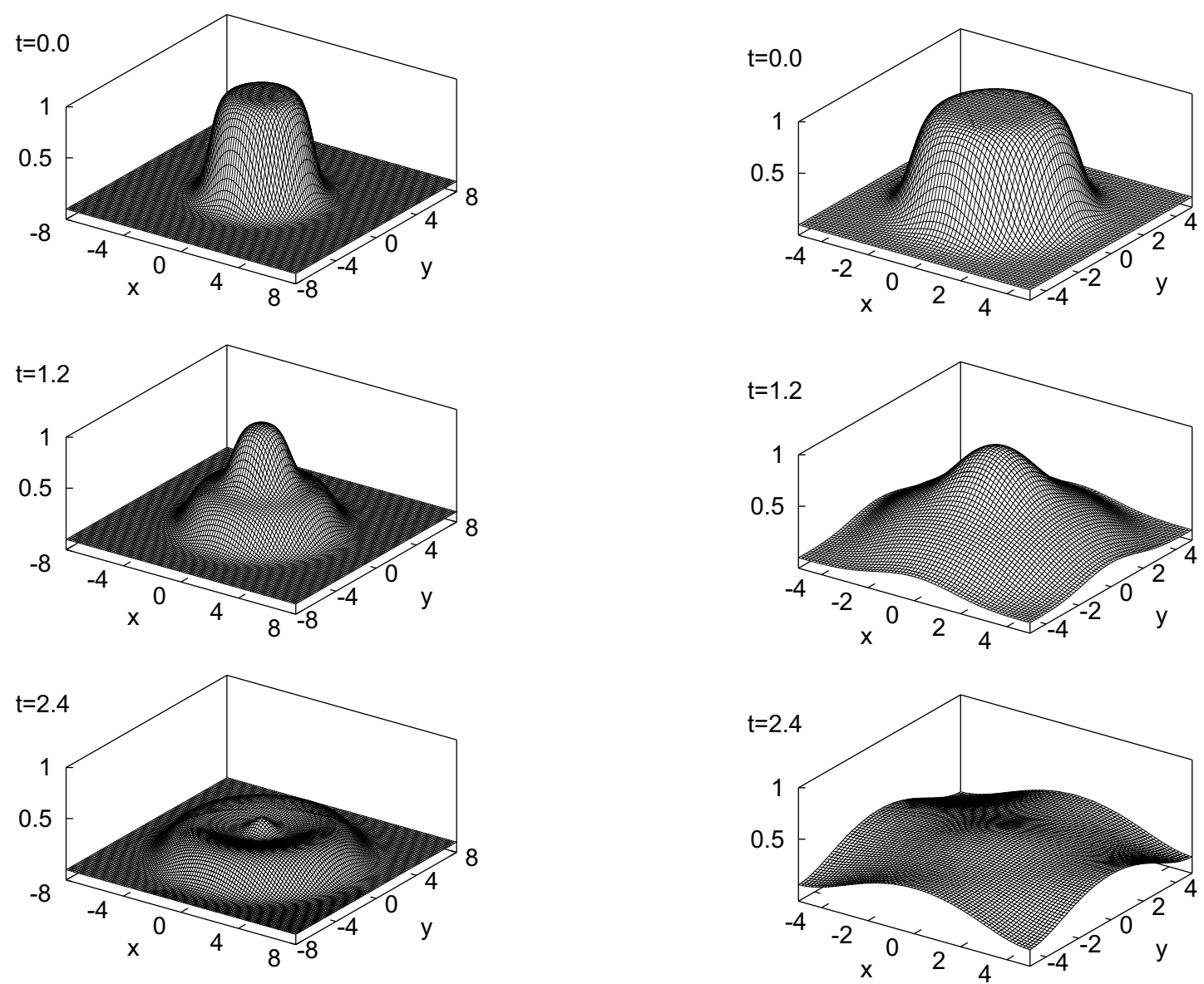

Fig. 4. Numerical solutions to the Volterra equation (1) in two spatial dimensions at chosen time steps: $\alpha=1.85$, closed boundary conditions.

different basis subspaces and the same grid size,

$$
\begin{aligned}
& \Delta u_{n, M}(t) \\
& \quad=\max \left|u_{n, M}^{\text {num }}\left(x_{i}, t\right)-u_{n-2, M}^{\text {num }}\left(x_{i}, t\right)\right|_{i=1}^{M},
\end{aligned}
$$

where $M=m^{d}$. In Fig. 7 the error estimate $\Delta u_{n, M}(t)$ is presented for the one-dimensional case $(d=1), \alpha=1.5$, grid size $m=121$ and $t=1.8$ in a semilogarithmic plot. The two-dimensional case $(d=2), m=101$ and the same values of $\alpha$ and $t$ is displayed in Fig. 8 . In Figs. 7 and 8 , the error estimates exhibit almost an exponential decrease with the growth of the basis subspace dimension $n$ in (3).

Comparing the approximate solutions for different grid sizes is a little more difficult because their values are given at different points. Therefore, in order to compare them, we define the error estimate in the following way:

$$
\begin{aligned}
& \Delta u_{n, m}(t) \\
& \quad=\max \left|u_{n, m}^{\mathrm{num}}\left(x_{i}, t\right)-\tilde{u}_{n, m^{\prime}}^{\mathrm{num}}\left(x_{i}, t\right)\right|_{i=1}^{M} .
\end{aligned}
$$

In (14), $\tilde{u}_{n, m^{\prime}}^{\text {num }}\left(x_{i}, t\right)$ stands for the value at the point of the bigger grid $m$ obtained from the solution on the smaller grid $m^{\prime}$ by cubic spline interpolation. Figure 9 shows an example of the decrease in the error estimate $\Delta u_{n, m}(t)$ for the dimension $d=2$. For the one-dimensional calculations (not shown here), the results exhibit the same behaviour. We see that for the fixed basis subspace the increase in the grid size leads to a substantial decrease in the error estimate. That decrease is, for larger values of $m$, close to an exponential decrease.

That almost exponential decrease in the error estimate as the function of the number of the basis functions (with a fixed grid) or as the the function of the grid size (with a fixed basis) suggests that in principle one can obtain the numerical solution with arbitrary precision choosing the appropriate basis and grid sizes. However, the bigger the basis and the grid size, the larger the number of the computer operations necessary to obtain the solution. To avoid the accumulation of round-off errors with an increase in computer operations, one needs to apply higher and higher precision (longer computer word), which 

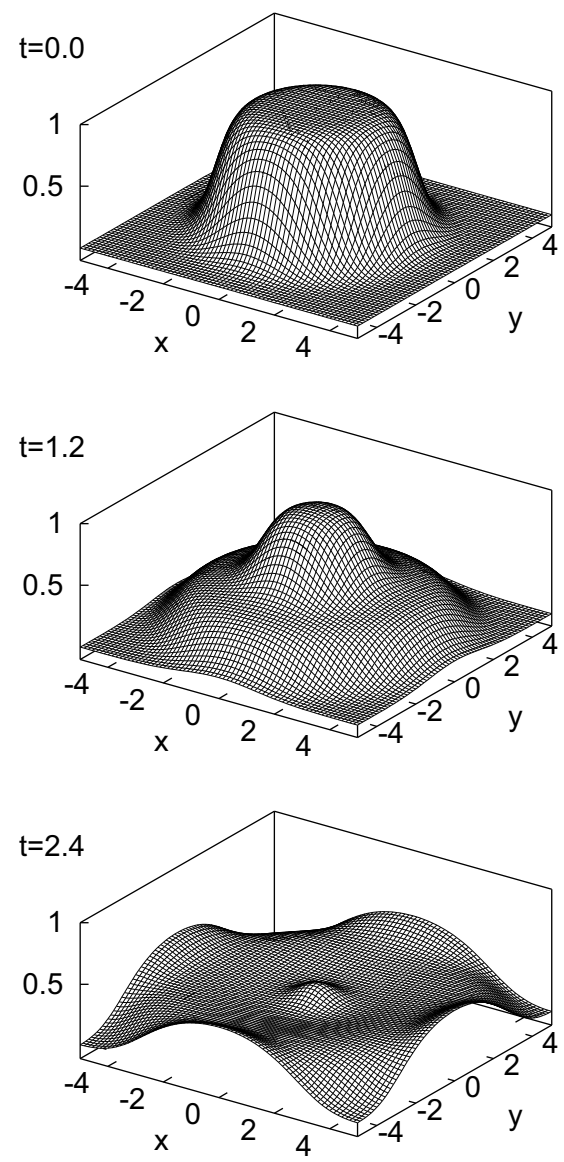

Fig. 6. Numerical solutions to the Volterra equation (1) in two spatial dimensions at chosen time steps: $\alpha=1.85$, periodic boundary conditions.

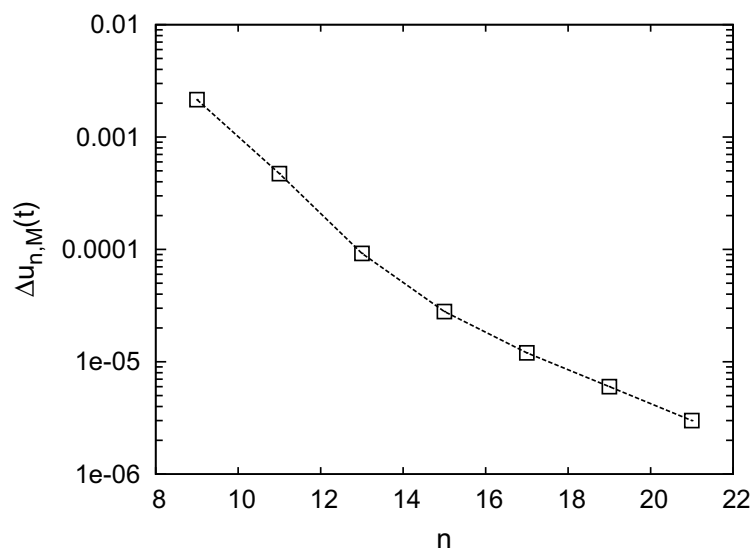

Fig. 7. Error estimate [13, $\Delta u_{n, M}(t)$ : the maximum difference between approximate solutions for which the number of basis functions differs by 2 as a function of $n$ for one spatial dimension $d=1$. The case $m=121, \alpha=1.5$ and $t=1.8$ is displayed.

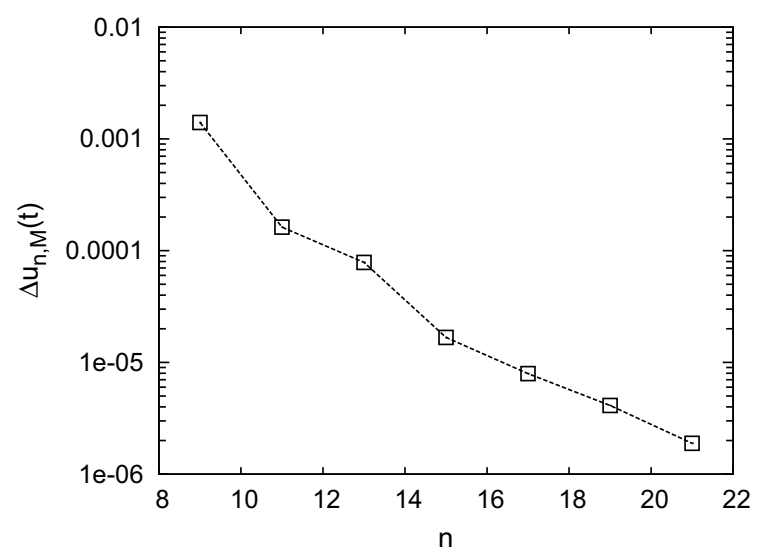

Fig. 8. Same as in Fig. 7 but for two spatial dimensions $d=2$ and grid size $m=101$.

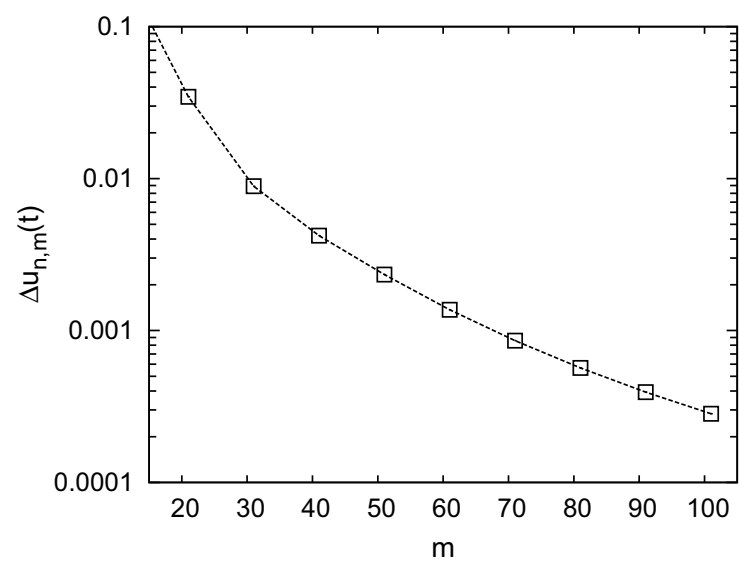

Fig. 9. Error estimate (14), $\Delta u_{n, m}(t)$ : the maximum difference between consecutive results obtained for grid sizes $m$ and $m^{\prime}=m-10$ in two spatial dimensions. The case $n=21$ basis functions, $\alpha=1.5$ and $t=1.8$ is presented.

results in much longer execution time and demands much more computer power. In practice, a reasonable compromise between the precision and computational effort is chosen for a given problem.

The results for the 3-dimensional case show similar qualitative behaviour. We do not present those here because the computing time grows substantialy. The case $d=3$ and $m=21$ requires the same order of computer operations as the case $d=2, m=101$ with the same $n$. The test computations made for grid sizes up to $m=21$ and the number of basis functions up to $n=20$ gave trends which qualitatively agree with those for $d=2$.

\section{Conclusions}

We presented a succesful numerical method of solving a class of Volterra equations (1) which are equivalent to dif- 
ferential equations with fractional time (2). From our results, the following conclusions can be drawn:

- For initial conditions spatially smooth enough and a reasonable choice of the $(n, m)$ parameters, the errors of the approximate numerical solution may be kept on a desired level.

- Our method works well for $d=1$ and $d=2$ spatial dimensions. However, the larger $d$, the more computer power is necessary. Test calculations indicate that the metod should work for $d=3$, too.

\section{References}

Barrett, R., Berry, M., Chan, T.F., Demmel, J., Donato, J., Dongarra, J., Eijkhout, V., Pozo, R., Romine, C. and der Vorst, H.V. (1994). Templates for the Solution of Linear Systems: Building Blocks for Iterative Methods, 2nd Edition, SIAM, Philadelphia, PA.

Bazhlekova, E. (2001). Fractional Evolution Equations in Banach Space, Ph.D. dissertation, Eindhoven University of Technology, Eindhoven.

Ciesielski, M. and Leszczyński, J. (2006). Numerical treatment of an initial-boundary value problem for fractional partial differential equations, Signal Processing 86(10): 26192631.

Fujita, Y. (1990). Integrodifferential equation which interpolates the heat equation and the wave equation, Osaka Journal of Mathematics 27(2): 309-321.

Gambin, Y., Massiera, G., Ramos, L., Ligoure, C. and Urbach, W. (2005). Bounded step superdiffusion in an oriented hexagonal phase, Physical Review Letters 94(11): 110602.

Goychuk, I., Heinsalu, E., Patriarca, M., Schmid, G. and Hänggi, P. (2006). Current and universal scaling in anomalous transport, Physical Review E 73(2): 020101 '(R)'.

Guermah, S., Djennoune, S. and Betteyeb, M. (2008). Controllability and observability of linear discrete-time fractionalorder systems, International Journal of Applied Mathematics and Computer Science 18(2): 213-222, DOI: 10.2478/v10006-008-0019-6.

Heinsalu, E., Patriarca, M., Goychuk, I. and Hänggi, P. (2009). Fractional Fokker-Planck subdiffusion in alternating fields, Physical Review E 79(4): 041137.

Heinsalu, E., Patriarca, M., Goychuk, I., Schmid, G. and Hänggi, P. (2006). Fokker-Planck dynamics: Numerical algorithm and simulations, Physical Review E 73(4): 046133.

Kaczorek, T. (2008). Fractional positive continuous-time linear systems and their reachability, International Journal of Applied Mathematics and Computer Science 18(2): 223-228, DOI: 10.2478/v10006-008-0020-0.
Kou, S. and Sunney Xie, X. (2004). Generalized Langevin equation with fractional Gaussian noise: Subdiffusion within a single protein molecule, Physical Review Letters 93(18): 180603 .

Labeyrie, G., Vaujour, E., Müller, C., Delande, D., Miniatura, C., Wilkowski, D. and Kaiser, R. (2003). Slow diffusion of light in a cold atomic cloud, Physical Review Letters 91(22): 223904.

Meltzer, R. and Klafter, J. (2000). The random walk's guide to anomalous diffusion: A fractional dynamics approach, Physics Reports 339(1): 1-77.

Ratynskaia, S., Rypdal, K., Knapek, C., Kharpak, S., Milovanov, A., Ivlev, A., Rasmussen, J. and Morfill, G. (2006). Superdiffusion and viscoelastic vortex flows in a twodimensional complex plasma, Physical Review Letters 96(10): 105010 .

Rozmej, P. and Karczewska, A. (2005). Numerical solutions to integrodifferential equations which interpolate heat and wave equations, International Journal on Differential Equations and Applications 10(1): 15-27.

Saad, Y. and Schultz, M. (1986). GMRES: A generalized minimal residual algorithm for solving nonsymmetric linear systems, SIAM Journal on Scientific and Statistical Computing 7(3): 856-869.

Schneider, W. and Wyss, W. (1989). Fractional diffusion and wave equations, Journal of Mathematical Physics 30(4): 134144.

Van der Vorst, H. (1992). Bi-CGSTAB: A fast and smoothly converging variant of Bi-CG for the solution of nonsymmetric linear systems, SIAM Journal on Scientific and Statistical Computing 13(2): 631-644.

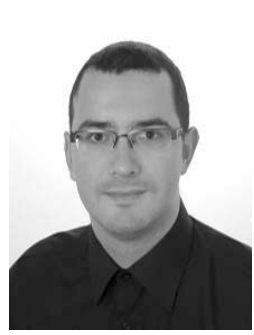

Bartosz Bandrowski is a $\mathrm{Ph} . \mathrm{D}$. student at the Faculty of Mathematics, Computer Science and Econometrics, University of Zielona Góra. He received an M.Sc. degree in mathematics from the University of Zielona Góra in 2008. His main interests include mathematical informatics, numerical methods in Volterra equations, fractional calculus and stochastic equations.

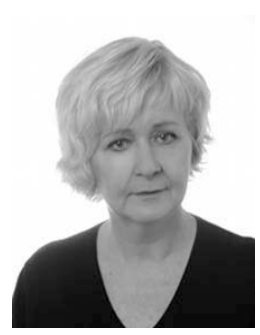

Anna Karczewska is an associate professor at the Faculty of Mathematics, Computer Science and Econometrics, University of Zielona Góra. She holds an M.Sc. degree from the Warsaw Technical University, a Ph.D. degree from Jagiellonian University, Cracow, and habilitation from the University of Zielona Góra. Her fields of interest include deterministic and stochastic integral equations, in particular Volterra equations of integral and fractional order, and fluid dynamics. 


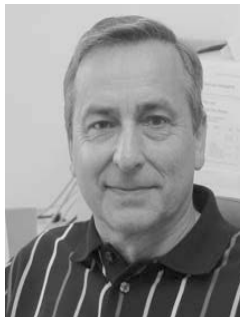

Piotr Rozmej is a professor at the Faculty of Physics and Astronomy, University of Zielona Gora, the head of the Division of Mathematical Methods in Physics. He holds an M.Sc. degree from Maria Curie-Skłodowska University, Lublin, a Ph.D. degree from Warsaw University, and habilitation from UMCS. He became a professor in 1992 and a full professor in 1997.

He has spent several years working as a senior researcher at the Joint Institute for Nuclear Research (Dubna, USSR), Gesellschaft für Schwerionenforschung (Darmstadt, Germany) and Institut des Sciences Nucléaires (Grenoble, France). He works in the fields of nuclear structure, nuclear reactions, properties of heavy and superheavy nuclei. Other areas of interest include quantum optics, in particular wave packet dynamics, and nonlinear dynamics.

Received: 24 May 2009

Revised: 28 September 2009 